\title{
Mining Billion-Node Graphs: Patterns, Generators and Tools
}

\author{
Christos Faloutsos \\ Computer Science Department, \\ Carnegie Mellon University \\ christos@cs.cmu.edu
}

What do graphs look like? How do they evolve over time? How to handle a graph with a billion nodes? We present a comprehensive list of static and temporal laws, and some recent observations on real graphs (like, e.g., "eigenSpokes"). For generators, we describe some recent ones, which naturally match all of the known properties of real graphs. Finally, for tools, we present "oddBall" for discovering anomalies and patterns, as well as an overview of the PEGASUS system which is designed for handling Billion-node graphs, running on top of the "hadoop" system. 